\title{
Simulations of the stopping efficiencies of fission ion guides
}

\author{
Andreas Solders ${ }^{1 \mathrm{a}}$, Ali Al-Adili ${ }^{1}$, Dmitry Gorelov ${ }^{2}$, Kaj Jansson ${ }^{1}$, Ari Jokinen ${ }^{2}$, Veli Kolhinen ${ }^{2}$, Mattias Lantz ${ }^{1}$, \\ Andrea Mattera ${ }^{1}$, Ian Moore ${ }^{2}$, Niklas Nilsson ${ }^{1}$, Martin Norlin $^{1}$, Heikki Penttilä ${ }^{2}$, Stephan Pomp ${ }^{1}$, Alexander V. Prokofiev ${ }^{1}$, \\ Vasileios Rakopoulos ${ }^{1}$, Sami Rinta-Antila ${ }^{2}$, Vasily Simutkin ${ }^{2}$, and the IGISOL group ${ }^{2}$ \\ 1 Uppsala University, Department of Physics and Astronomy, PO Box 256, 75105 Uppsala, Sweden \\ ${ }^{2}$ University of Jyväskyl”a, Department of Physics, PO Box 35, 40014 University of Jyväskylä, Finland
}

\begin{abstract}
With the Ion Guide Isotope Separator On-Line (IGISOL) facility, located at the University of Jyväskylä, products of nuclear reactions are separated by mass. The high resolving power of the JYFLTRAP Penning trap, with full separation of individual nuclides, capacitates the study of nuclides far from the line of stability. For the production of neutron-rich medium-heavy nuclides, fissioning of actinides is a feasible reaction. This can be achieved with protons from an in-house accelerator or, alternatively, with neutrons through the addition of a newly developed $\mathrm{Be}(\mathrm{p}, \mathrm{xn})$-converter. The hereby-obtained fission products are used in nuclear data measurements, for example fission yields, nuclear masses, Q-values and decay spectroscopy.

Prior to separation, the ionized reaction products are stopped in a helium-filled gas cell, referred to as the ion-guide. In this work we present simulations of the stopping of fission products in an ion guide developed for neutron-induced fission. The production and extraction rates are evaluated and compared against experimental values.
\end{abstract}

\section{Introduction}

Independent fission product yields are an important characteristic of nuclear fission and better knowledge of how the yield distributions varies with fissioning system will improve our understanding of the fission process. For energy applications fission yield data are important in criticality and reactivity calculations for reactor design, dosimetry and fission gas production for reactor safety and for improved burn-up predictions [1,2]. For the management of nuclear waste, whether it will be repositories, reprocessing, transmutation or Generation IV reactors, good knowledge of the composition of the spent fuel is required. The inventory of fission products also dominates the decay heat the first $50-80$ years after extraction of spent nuclear fuel from a reactor [3].

At the physics laboratory of the University of Jyväskylä, independent fission product yields have been measured for proton induced fission of ${ }^{n a t} \mathrm{U}$ using the Ion Guide and Isotope Separator On-Line (IGISOL) technique [4]. Using this technique, fission is induced in a target situated in a helium gas filled container, called an ion guide. Through collisions with the helium gas the highlyionized high-energy fission products are recombined to form singly charged ions and are brought to rest. A helium gas flow, in combination with a series of electrostatic electrodes, are used to extract the products from the ion guide. Using a dipole magnet, a single isobaric chain is selected and transported to the Penning trap, JYFLTRAP, where isobaric separation based on mass is performed at a resolving power of more than $10^{5}$. Hence, with this method independent fission yields can be measured through direct ion counting just a few hundred milliseconds after fission.

a e-mail: andreas.solders@physics.uu.se
For more details on the experimental method see Penttilä et al. [4].

For nuclear energy applications neutron induced fission is of main interest. Neutron induced fission also produces more neutron rich products, of interest in the studies of nuclides far from the line of stability. To facilitate this reaction at IGISOL a $\mathrm{Be}(\mathrm{p}, \mathrm{xn})$ neutron converter and a dedicated ion guide for neutron induced fission has been developed [5-7].

\section{Method}

The fission process produces a wide range of nuclides with atomic mass numbers ranging from 70 to 160. In addition, fragments show a large spread in kinetic energy, from 0.3 to $1.2 \mathrm{MeV} / \mathrm{u}$. This could potentially lead to systematic effects in fission yield measurements due to differences in the stopping efficiencies of the fission fragments. In order to investigate this, the ion guide for proton induced fission was studied in simulations and the the efficiency was calculated as a function of mass, charge number and energy of the fission fragments. The study was performed using a combination of the Monte Carlo codes GEF [8], Geant4 [9] and SRIM [10], and the results show that, with a proper combination of helium pressure and geometrical properties of the ion guide, systematic effects in the stopping power can be negligible [11].

The geometrical properties of the ion guide, as well as the properties of the fission fragments, for the neutron induced setup differs from the proton-induced case. Therefore, a new framework that can simulate the whole process, from proton beam to extracted fission product, has been developed. 


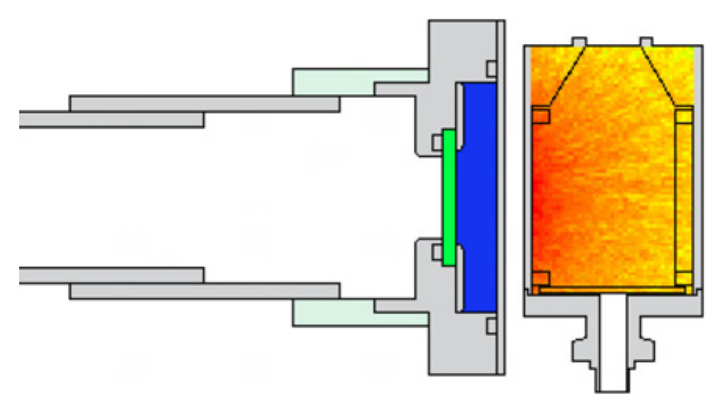

Figure 1. Cross sectional view of the neutron converter and ion guide of the MCNPX model. The proton beam enters from left. The beryllium disc is indicated in green with the cooling water behind. The Uranium targets are mounted in a holder close to the wall of the ion guide, facing the neutron converter. The recorded neutron flux in the ion guide is displayed in color scale.

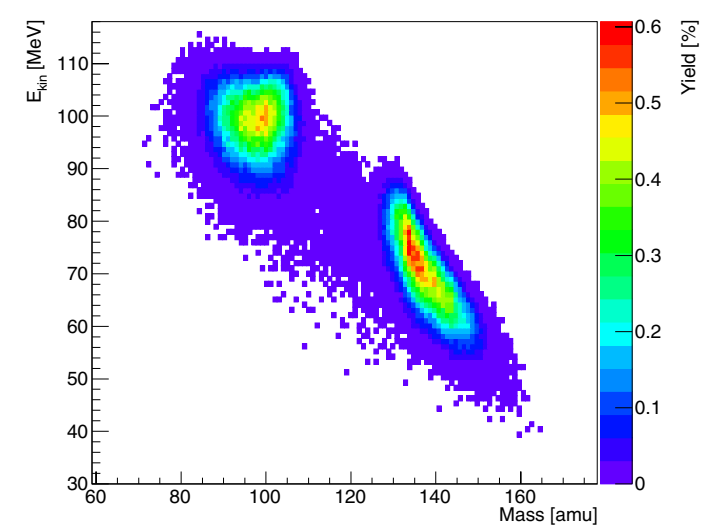

Figure 2. GEF simulation of fission fragment yields of ${ }^{238} \mathrm{U}(\mathrm{n}, \mathrm{f})$ at $\mathrm{E}_{n}=4 \mathrm{MeV}$ as a function of fragment kinetic energy and mass.

\section{Simulations}

The purpose of this work is to create a complete simulation framework of the neutron converter and ion guide from the incoming proton beam to the extracted fission products. The result can be used to optimize the geometry with respect to production rate, while at the same time minimizing systematic effects. To this end, a combination of different Monte Carlo codes have been used. The production of neutrons in the $\mathrm{Be}(\mathrm{p}, \mathrm{xn})$ converter was simulated using MCNPX 2.5.0 [12] and the neutron field in the volume occupied by the ion guide was extracted. The neutron induced fission process, including multi chance fission, was handled by the GEF code while a Geant 4 model was developed for the transportation and stopping of fission products in the ion guide. Finally, the multiphysics code COMSOL $\mathrm{R}$, was used to study the evacuation of fission products from the ion guide.

\subsection{MCNPX}

The MCNPX model includes the ion guide together with the $5 \mathrm{~mm}$ thick beryllium plate in its aluminum holder with cooling water behind. The holder is connected to the aluminum beam tube through a plastic insulator and an aluminum extension pipe. Everything sits inside the target chamber of the IGISOL setup. A $30 \mathrm{MeV}$ proton beam is impinged on the beryllium target and the resulting neutron flux is recorded using a rectangular $3 \mathrm{D}$ grid covering the ion guide. The spatial resolution of the grid is $1 \mathrm{~mm}^{3}$ and

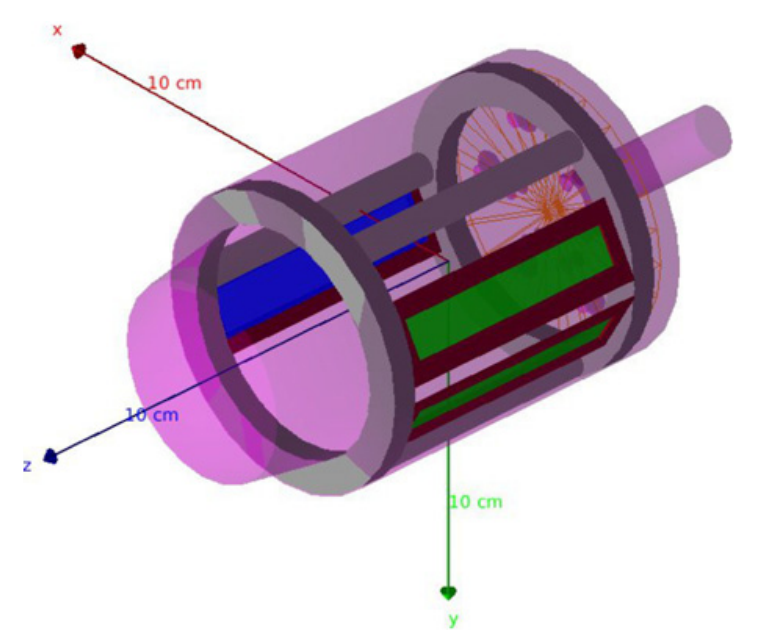

Figure 3. Geometry used in the Geant 4 calculations. Inside the ion guide (pink) is an aluminum holder (gray). The two uranium targets (green) are mounted in the holder together with an implantation foil (blue). The neutron field propagates along the $\mathrm{x}$-axis while the fission products are extracted in the direction of the z-axis.

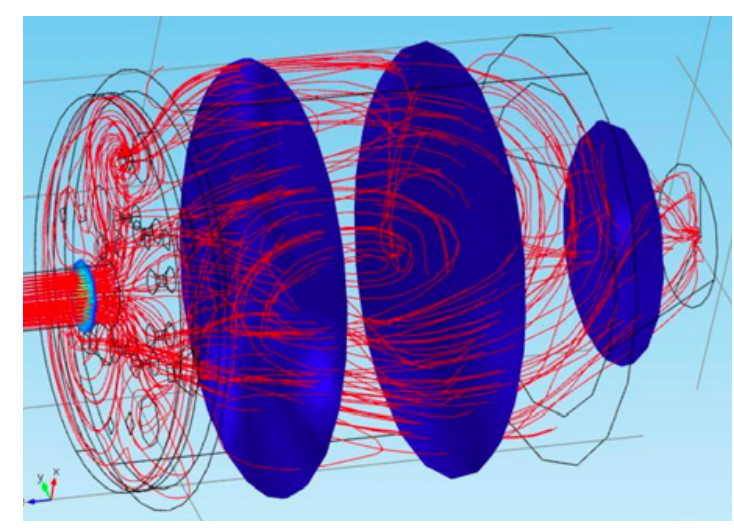

Figure 4. COMSOL $®$ multiphysics model of the helium gas flow in the ion guide. The lines represent the paths of imaginary particles carried by the flow.

the flux was recorded in energy bins of $1 \mathrm{MeV}$. A cross sectional view of the converter and ion guide can be seen in Fig. 1. From the neutron flux, a fission rate is calculated in each grid point.

\subsection{GEF}

The General Description of Fission Observables (GEF) code [8], version 2015/2.1, has been used to obtain the yields and kinetic energies of the fission products (Fig. 2). In our previous work [11] 14 evenly spaced masses were selected to cover the full mass range, and for each mass five characteristic energies were studied. In the present work, however, a GEF calculation has been made for every neutron energy from 1 to $30 \mathrm{MeV}$ in steps of $2 \mathrm{MeV}$. The energy of the fission product has then been sampled uniformly from the full energy range and the result has been weighted with the neutron-energy dependent yielddistribution.

\subsection{Geant4}

Geant4 is used to simulate the stopping of fission products in the helium gas. From earlier simulations [11] it is known 

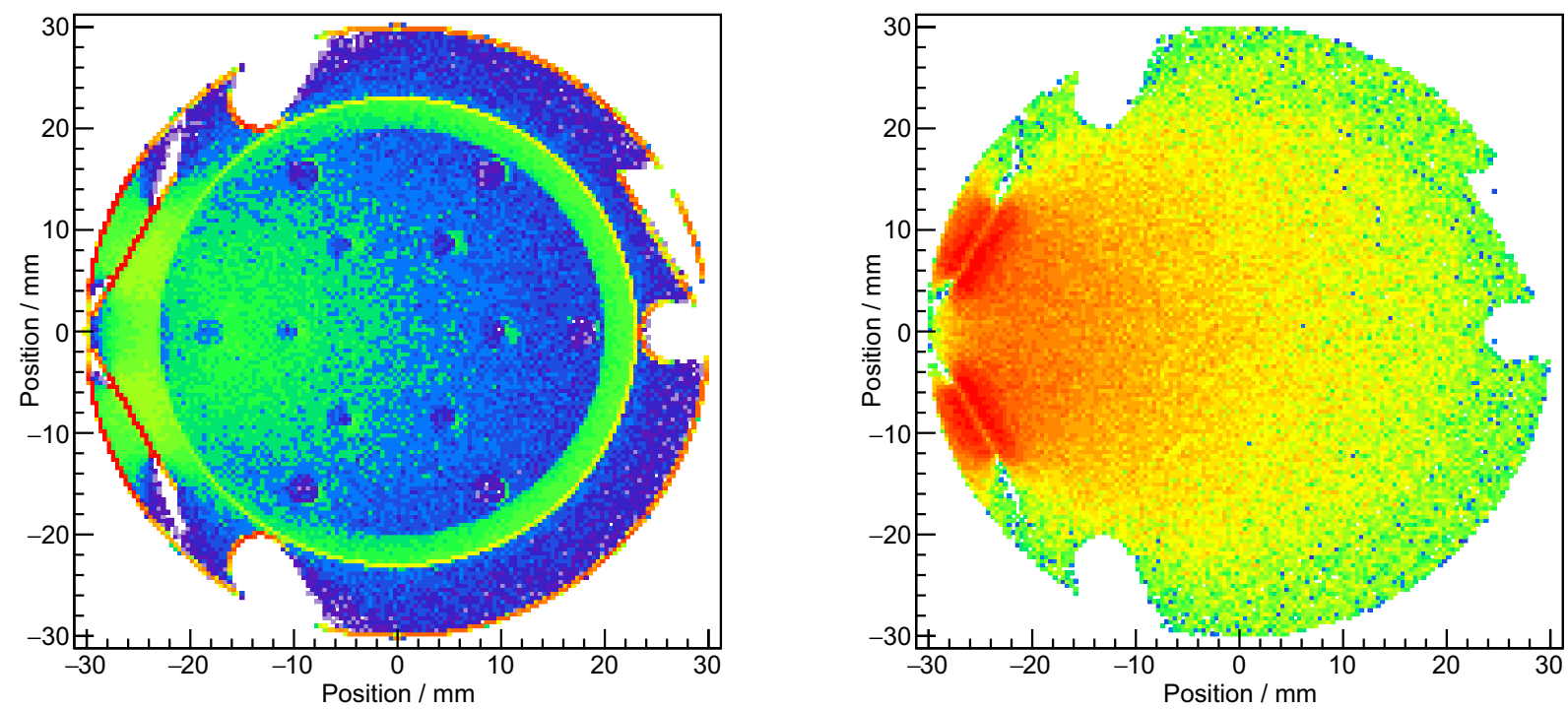

Figure 5. Stopping of the fission product ${ }^{112} \mathrm{Rh}$ in the ion guide. The left figure shows the final position of all ions while the right figure only includes ions that have come to a stop in the helium gas. Both figures are projections onto the xy-plane.

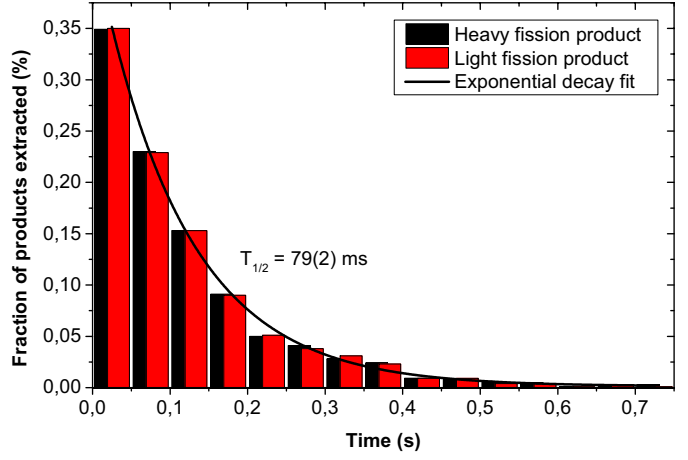

Figure 6. Histogram of evacuation times from the

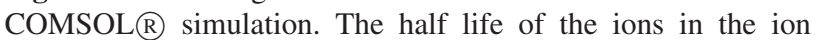
guide of $79 \mathrm{~ms}$ correspond to a mean evacuation time of $113 \mathrm{~ms}$. These numbers seem to be independent of ion mass.

that in order for a fission product to stop in the gas it must loose most of its energy already in the uranium target, before entering the helium gas. The starting positions of the fission products are sampled uniformly within the two uranium targets and are later weighted with the fission rate at the corresponding position.

The geometry of the Geant 4 model is shown in Fig. 3. The targets, marked in green in the figure, are mounted in two separate frames that face the neutron converter. The frames are kept in place using an aluminum holder that also holds an implantation foil. In order to bench mark the simulations, the number of fission products that stop in this foil will be compared to gamma spectroscopy of a real foil used in experiment.

\subsection{COMSOL $R$}

After the fission products come to rest inside the ion guide they are extracted by the flow of helium gas. To access the extraction efficiency and extraction time of fission products from the ion guide a COMSOL $\AA$ multiphysics model has been developed. The nominal inlet helium pressure is set to 200 mbar while the target chamber, on the other side of a $1.2 \mathrm{~mm}$ outlet aperture, is at vacuum. At the inlet the helium gas is distributed in the ion guide volume using a diffuser. The helium gas flow lines generated by COMSOL $\AA$ are shown in Fig. 4. Although some turbulence can be observed the flow is mostly laminar.

\section{Results}

The development of this simulation framework is still ongoing and only preliminary results are reported here. From the MCNPX simulation the average neutron flux in the two targets is $2.2 \cdot 10^{9}$ neutrons per second at $1 \mu \mathrm{A}$ proton beam. This will result in a total fission rate of $5.5 \cdot 10^{5}$ fissions per second using two standard IGISOL targets of $10 \mathrm{~mm}$ by $50 \mathrm{~mm}$ at a thickness of $14 \mathrm{mg} / \mathrm{cm}^{2}$. So far, the full simulation has only been completed for a few fission products and here we will focus on the results for ${ }^{112} \mathrm{Rh}$. This is an important case since ${ }^{112} \mathrm{Rh}$ is regularly used at IGISOL for beam optimization, measuring the beta activity after isobaric separation.

In Fig. 5 the result for ${ }^{112} \mathrm{Rh}$ is presented. The left part of the figure shows the final position, when coming to rest, of all of the Rhodium ions. This confirms earlier simulations which show that most ions never exit the uranium targets and of those that do, a large fraction has too large energy to stop in the gas and instead gets implanted in the wall of the ion guide.

The right part of Fig. 5 shows the final position of the ions that stop in the gas. Most of the ions that end up in the gas are stopped close to the uranium targets. The total number of ${ }^{112} \mathrm{Rh}$ ions stopped in the gas is 24 per second at a proton beam current of $1 \mu \mathrm{A}$, which amounts to about $2.3 \%$ of the total number of ${ }^{112} \mathrm{Rh}$ produced in the targets.

To obtain information about the extraction efficiency and extraction times of the ion guide from the

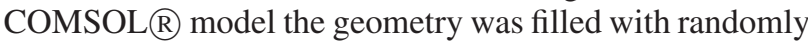
distributed charged particles. The physics were set to let the particles interact with the metal surfaces and get stuck whenever in contact. The result shows that the helium flow is sufficient to extract about $15 \%$ of the ions and the average extraction time of those that get 
out is $113 \mathrm{~ms}$ (Fig. 6). These numbers are in reasonable agreement with earlier estimates which suggest that the total extraction efficiency, including the subsequent electrostatic extraction, is of the order of $10 \%$, and that the evacuation time of the ion guide is about $250 \mathrm{~ms}$ [7]. A comparison has also been made between the extraction times of light and heavy fission products which show no significant differences (Fig. 6).

Putting all together we can, at least preliminary, conclude that the count rate of mass separated ${ }^{112} \mathrm{Rh}$ ions after the dipole magnet will probably be as low as one, or a few, per second at $1 \mu \mathrm{A}$ proton beam current. In proton-induced fission, count rates of several thousand ${ }^{112} \mathrm{Rh}$ per second can be obtained, however, the yield of ${ }^{112} \mathrm{Rh}$ is about ten times higher in proton induced fission compared to the neutron induced case. Never the less, the MCC 30 cyclotron at Jyväskylä could potentially deliver up to $100 \mu \mathrm{A}$ of proton beam which would give a reasonable count rate for ${ }^{112} \mathrm{Rh}$ in neutron induced fission.

\section{Outlook}

Preparations are being made for a neutron-induced fission run at IGISOL using the neuron converter and ion guide. The neutron field will be measured and compared to the simulations using Thin Film Breakdown Counters [13] in Time Of Flight-mode. The production rate of fission products will be measured through the activity induced in the implantation foil, mounted on the opposite side of the ion guide with respect to the targets.

The next step will be to run the simulations with ions covering the the full mass range of fission products. For each mass the element with the highest yield will be used. The purpose is to investigate whether or not there is a mass dependence in the stopping of the products, that can cause systematic errors in measured mass yields. If such a dependence is observed it can be characterized using simulations in combination with calibration measurements. The simulation framework described in this paper will also be used to study the behavior of ion guides of different sizes and at different helium pressures.

We would like to acknowledge the Swedish Radiation Safety Authority (SSM) and the Swedish Nuclear Fuel and Waste Management Company (SKB) for their financial support.

\section{References}

[1] J. Blachot, et al., IAEA-TECDOC 1168 (2001)

[2] P. Dimitriou, et al., IAEA-INDC(NDS) 0713 (2016)

[3] R. Mills and D. Parker, AIP Conference Proceedings 769, 136 (2005)

[4] H. Penttilä, et al., The European Physical Journal A 52, 104 (2016)

[5] A. Solders, et al., Nuclear Data Sheets 119, 338 (2014)

[6] A. Mattera, et al., Nuclear Data Sheets 119, 416 (2014)

[7] D. Gorelov, et al., Nuclear Instruments and Methods in Physics Research Section B 376, 46 (2016)

[8] K.H. Schmidt, et al., Nuclear Data Sheets 131, 107 (2016)

[9] S. Agostinelli et al., Nuclear Instruments and Methods in Physics Research Section A 506, 250 (2003)

[10] J. Ziegler, M.D. Ziegler, J. Biersack, Nuclear Instruments and Methods in Physics Research Section B 268, 11 (2010)

[11] A. Al-Adili, et al., The European Physical Journal A 51, 59 (2015)

[12] D.B. Pelowitz, Ed., MCNPX Users Manual Version 2.5.0", LA-CP-05-0369 (2005)

[13] A.N. Smirnov, et al., Nuclear Instruments and Methods in Physics Research Section A 687, 14 (2012) 